\title{
THE SIGNIFICANCE OF EMERGING PROPERTY SECTORS IN PROPERTY PORTFOLIOS
}

\author{
GRAEME NEWELL and HSU WEN PENG \\ University of Western Sydney
}

\begin{abstract}
Property funds in Australia have over $\$ 160$ billion in assets, with office, retail and industrial property being the major property sectors contributing to these property portfolios. However, recent years have seen increased attention given to the property investment opportunities available from the emerging property sectors such as self-storage, healthcare, retirement facilities and leisure/entertainment. This paper will assess the significance of these emerging property sectors in property portfolios in Australia; particularly highlighting issues such as the current portfolio levels and the leading property funds in these emerging property sectors. An emerging sector LPT performance index is established and an emerging property sector risk-adjusted performance analysis carried out over 2002-2005, as well as the portfolio diversification benefits of the emerging property sector assessed. Compared to the other LPT sectors, the emerging sector LPTs were seen to provide superior risk-adjusted performance, as well as providing portfolio diversification benefits.
\end{abstract}

Keywords: Emerging property sectors, property portfolios, property vehicles, self-storage, healthcare, retirement facilities, leisure/entertainment, emerging sector performance index, performance analysis.

\section{INTRODUCTION}

The emerging property sectors, such as self-storage, retirement facilities and leisure/entertainment, have taken on increased importance in property portfolios in recent years. This paper assesses the context for this increased importance, the significance of the emerging property sectors in property portfolios in Australia, as well as assessing the added-value of the emerging property sectors using risk-adjusted performance analysis over 2002-2005.

Institutional investors in Australia have largely traditionally concentrated on low-risk core property portfolios of office, retail and industrial properties, with non-core properties accounting for less than 5\% of these institutional portfolios (Higgins, 2005). This sees Australian property funds having $\$ 160$ billion in direct property assets (PIR, 2004); for 
example, significant property portfolios include Westfield (\$32 billion), Colonial First State (\$21 billion), AMP Capital (\$14 billion) and Macquarie (\$10 billion) (PIR, 2004).

However, recent years have seen significant capital inflows available for property (eg: growth in superannuation fund assets), significant growth in LPTs, a shortage of quality local commercial properties and subsequent reduced yields (Blundell, 2005b). These drivers have seen LPTs seek international property investment opportunities (eg: US retail, US industrial, European retail) (Blundell, 2005a, 2005c; Larsen, 2005; Murdoch, 2004; Tan, 2004a, b), with international property currently accounting for $35 \%$ of LPT property assets (DB RREEF, 2005; Oliver, 2004), and these levels expected to increase to 50-60\% by 2009 (Norris, 2004). LPTs have also become increasingly involved in non-property investment activities such as property development via stapled securities structures (Oliver, 2004; Tan, 2004c).

This mismatch between available funds and available core property assets in Australia has also seen institutional investors expand their focus beyond these traditional property sectors to consider both higher risk value-added property and opportunistic property (DB RREEF, 2005; Lowrey, 2005; Schuck and Howard, 2005). This has seen increased attention given to the property investment opportunities for enhanced returns available from the emerging property sectors, such as self-storage, healthcare, retirement, carparks, and leisure and entertainment (Blundell, 2003, 2004, 2005b, c; DB RREEF, 2005; Larsen, 2003, 2005) and from the property-related sectors such as infrastructure (Blundell, 2005a; DB RREEF, 2005). The retirement and healthcare sectors have also received additional institutional attention due to the demographic shift with the ageing population, which will see the population percentage over 65 years in Australia increase from 13\% in 2006 to nearly $26 \%$ by 2045 (ABS, 2004; Blundell, 2003, 2004; Larsen, 2003).

Whilst only recently being considered in Australia, these emerging or non-core property sectors have been widely utilised by US REITs over the last ten years (Blundell, 2005b; NAREIT, 2005a, b). At September 2005, the US emerging sectors of healthcare, selfstorage and speciality comprised 23 equity REITs and accounted for over US\$43 billion in market capitalisation, representing $14.5 \%$ of the equity REIT market (see Table 1) (NAREIT, 2005a, b). Some of these emerging sector REITs are amongst the largest US REITs (see Table 2); namely Public Storage (US\$8.7 billion; 7th largest US REIT), Plum Creek Timber (US\$ 7.0 billion; 11th largest) and HealthCare Property (US\$3.6 billion; 21 st largest) (NAREIT, 2005a, b). Amongst these speciality REITs, the property sectors include timberland, communication tower sites, automotive retail property, movie theatre complexes, prisons and railways. In comparison, the largest traditional sector US REITs were Simon Property Group (retail; US\$16.4 billion), Equity Office Properties Trust (office; US\$13.3 billion) and Vornado Realty Trust (diversified; US\$12.2 billion) (NAREIT, 2005a, b). 
Table 1: Significance of emerging property sectors in US equity REITs: September 2005

\begin{tabular}{lccc}
\hline REIT sector & $\begin{array}{c}\text { Number of } \\
\text { REITs }\end{array}$ & $\begin{array}{c}\text { Market } \\
\text { capitalisation } \\
\text { (US\$) }\end{array}$ & $\begin{array}{c}\text { Percentage in } \\
\text { equity REIT index }\end{array}$ \\
\hline Office & 24 & $\$ 56.5 \mathrm{~B}$ & $18.8 \%$ \\
Retail & 33 & $\$ 83.2 \mathrm{~B}$ & $27.7 \%$ \\
Industrial & 7 & $\$ 20.4 \mathrm{~B}$ & $6.8 \%$ \\
Mixed office/ind. & 7 & $\$ 10.5 \mathrm{~B}$ & $3.5 \%$ \\
Diversified & 14 & $\$ 20.1 \mathrm{~B}$ & $6.7 \%$ \\
Residential & 26 & $\$ 48.8 \mathrm{~B}$ & $16.3 \%$ \\
Hotel & 18 & $\$ 17.6 \mathrm{~B}$ & $5.8 \%$ \\
Healthcare & 11 & $\$ 15.3 \mathrm{~B}$ & $5.1 \%$ \\
Self-storage & 5 & $\$ 13.3 \mathrm{~B}$ & $4.4 \%$ \\
Specialty & 7 & $\$ 15.0 \mathrm{~B}$ & $5.0 \%$ \\
\hline Total & 152 & $\$ 300.6 \mathrm{~B}$ & $100.0 \%$ \\
\hline Soure: & & &
\end{tabular}

Source: NAREIT $(2005 a, b)$ 
Table 2: Major US emerging sector REITs: September 2005

REIT sector

Market capitalisation (US\$)

Self-storage REITs

Public Storage

$\$ 8.7 \mathrm{~B}$

Shurgard Storage

$\$ 2.6 \mathrm{~B}$

Sovran Self-storage

$\$ 0.8 \mathrm{~B}$

U-Store-It Trust

$\$ 0.8 \mathrm{~B}$

Extra Space Storage

$\$ 0.5 \mathrm{~B}$

Healthcare REITs

HealthCare Property

$\$ 3.6 \mathrm{~B}$

Ventas

$\$ 3.3 \mathrm{~B}$

Health Care REIT

$\$ 1.9 \mathrm{~B}$

Healthcare Realty Trust

$\$ 1.9 \mathrm{~B}$

Nationwide Health Properties

$\$ 1.5 \mathrm{~B}$

Senior Housing Properties Trust

\$1.3B

National Health Investors

$\$ 0.8 \mathrm{~B}$

Omega Healthcare Investors

$\$ 0.7 \mathrm{~B}$

LTC Properties

$\$ 0.5 \mathrm{~B}$

Universal Health Realty Income Trust

$\$ 0.4 \mathrm{~B}$

Medical Properties Trust

$\$ 0.4 \mathrm{~B}$

Windrose Medical Properties Trust

$\$ 0.2 \mathrm{~B}$

National Health Realty

$\$ 0.2 \mathrm{~B}$

Specialty REITs

Plum Creek Timber Company

$\$ 7.0 \mathrm{~B}$

Global Signal

$\$ 3.1 \mathrm{~B}$

Rayonier

$\$ 2.9 \mathrm{~B}$

Capital Automotive REIT

$\$ 1.8 \mathrm{~B}$

Entertainment Properties Trust

$\$ 1.1 \mathrm{~B}$

Correctional Properties Trust

$\$ 0.3 \mathrm{~B}$

Pittsburgh \& West Virginia Rail Road

$\$ 0.1 \mathrm{~B}$

Source: NAREIT (2005a, b)

180

Pacific Rim Property Research Journal, Vol 12, No 2 
The performance of the US emerging sector equity REITs over 1994-2005 is given in Table 3, with self-storage REITs seen to be the second best REIT sector on a risk-adjusted performance basis, as shown by the Sharpe index. The risk levels for these emerging sector REITs were above the risk level for the overall equity REIT sector and above the risk level for most of the traditional sector REITs. The US emerging sector REITs were also less highly correlated than the traditional sector REITs with the overall equity REIT sector, reflecting within-REIT sector portfolio diversification benefits, as well as the emerging sector REITs not being highly correlated with stocks (see Table 4), reflecting portfolio diversification opportunities. These analyses confirm the investment performance of these US emerging sector REITs; particularly compared to the traditional sector REITs.

Table 3: US emerging sector equity REIT performance: Q1: 1994 - Q3: 2005

\begin{tabular}{lccc}
\hline REIT sector & $\begin{array}{c}\text { Average } \\
\text { annual return }\end{array}$ & Annual risk & Sharpe index $^{(\mathbf{1})}$ \\
\hline Office & $16.7 \%$ & $15.20 \%$ & $0.85(4)$ \\
Retail & $16.6 \%$ & $14.10 \%$ & $0.91(3)$ \\
Industrial & $17.1 \%$ & $13.95 \%$ & $0.95(1)$ \\
Residential & $13.5 \%$ & $12.80 \%$ & $0.76(5)$ \\
Diversified & $11.9 \%$ & $15.42 \%$ & $0.53(7)$ \\
Hotel & $6.8 \%$ & $27.36 \%$ & $0.11(9)$ \\
Healthcare & $13.7 \%$ & $\mathbf{1 9 . 0 9 \%}$ & $\mathbf{0 . 5 2}(\mathbf{8})$ \\
Self-storage & $\mathbf{1 7 . 7 \%}$ & $\mathbf{1 4 . 7 3 \%}$ & $\mathbf{0 . 9 5}(\mathbf{2})$ \\
Specialty & $\mathbf{4 . 9 \%}$ & $\mathbf{2 2 . 0 1 \%}$ & $\mathbf{0 . 0 5}(\mathbf{1 0})$ \\
\hline Total & $13.7 \%$ & $13.43 \%$ & $0.74(6)$
\end{tabular}

Source: Authors' calculations from NAREIT (2005a, b)

(1) Ranks based on risk-adjusted performance are given in brackets. 
Table 4: US emerging property sector REIT correlations: Q1: 1994 - Q3: 2005

\begin{tabular}{lcccccccc}
\hline & Total & $\begin{array}{c}\text { Self- } \\
\text { storage }\end{array}$ & Healthcare & Specialty & Office & Retail & Industrial & Stocks \\
Total & 1.00 & & & & & & & \\
Self-storage & 0.79 & 1.00 & & & & & & \\
Healthcare & 0.77 & 0.74 & 1.00 & & & & \\
Specialty & 0.58 & 0.48 & 0.31 & 1.00 & & & \\
Office & 0.88 & 0.71 & 0.61 & 0.52 & 1.00 & & & \\
Retail & 0.89 & 0.70 & 0.77 & 0.53 & 0.64 & 1.00 & & \\
Industrial & 0.88 & 0.62 & 0.61 & 0.43 & 0.77 & 0.79 & 1.00 & \\
Stocks & 0.32 & 0.25 & 0.07 & 0.47 & 0.42 & 0.15 & 0.18 & 1.00 \\
\hline \multicolumn{2}{l}{ Source: Authors' calculations from NAREIT $(2005 \mathrm{a}, \mathrm{b})$} & & & & \\
\hline
\end{tabular}

Source: Authors' calculations from NAREIT (2005a, b)

Only limited research has been conducted regarding these US emerging sector REITs; typically for self-storage (MiniCo, 2005; Severino, 2005) and seniors housing (Lowrey, 2005). Similarly, in Australia, emerging sector research has only been a general property industry commentary (eg: Blundell, 2003, 2004, 2005 a,b,c; Larsen, 2002, 2005), addressing industry-specific issues (eg: self-storage) (Blackwell, 2005; Kennard and Blackwell, 2005; SSAA, 2005) or the role of emerging property sectors in a property portfolio (DB RREEF, 2005; Schuck and Howard, 2005). Importantly, these emerging property sectors have some different key features to the traditional property sectors for institutional investors to assess in formulating their property portfolio strategies, including the operating business being linked with the property assets, difficulties predicting cashflows, a lack of consistent and long-term performance measures, small size of these niche markets, lack of institutional experience with emerging property sectors, the need for revised fund mandates to invest in these emerging property sectors, and whether these emerging sectors should be regarded as "property" or "property-related" (Blundell, 2003, 2004, 2005b; DB RREEF, 2005; Larsen, 2003; Schuck and Howard, 2005).

As such, the purpose of this paper is to rigorously assess the significance of these emerging property sectors in property portfolios in Australia; particularly highlighting issues such as the current portfolio levels and the leading property funds in the emerging property sectors. An emerging sector LPT performance index is established and an emerging property sector risk-adjusted performance analysis carried out over 2002-2005, as well as the portfolio diversification benefits of the emerging property sectors assessed. 


\section{METHODOLOGY}

\section{Emerging property sector funds profile}

Over 580 individual direct property funds in Australia were reviewed to assess the significance of the emerging property sectors in these funds. Property funds were identified from PIR (2004a), as well as from recent annual reports and product disclosure statements (PDS). Details determined per emerging property sector fund were emerging sector, year established, fund type, number of properties, total assets and fund manager. This resulted in 69 emerging sector funds being identified across seven emerging property sectors, including self-storage (8 funds), retirement (12), childcare (7), leisure/entertainment (13), healthcare (12), carparks (4) and agriculture (13). Hotels and residential property funds were not included in this study. ${ }^{(1)}$

\section{Emerging property sector performance analysis}

Monthly total returns were obtained from UBS (2005) for the three year period of November 2002 - October 2005 for the emerging sector LPTs listed on the ASX. Of the nine currently listed emerging sector LPTs, five have monthly returns over this three year period; namely Macquarie Leisure, Tourism and Leisure, MTM Entertainment, MFS Living and Leisure, Challenger Wine. Risk-adjusted performance analysis was carried out for each of these emerging sector LPTs, and compared to a number of traditional sector individual LPTs (eg: Stockland, GPT).

To assess the overall significance of the emerging sector LPTs, market cap-weighted and asset value-weighted emerging sector LPT performance indices were established using these five emerging sector LPTs available over this three year period of 2002-2005. The risk-adjusted performance analysis for the emerging sector LPTs was carried out and compared to the other LPT sectors (eg: office, retail, industrial etc) and the overall stockmarket. The inter-LPT sector correlations were used to assess the portfolio diversification benefits of the emerging sector LPTs.

\section{EMERGING SECTOR FUND PROFILE}

Table 5 presents the Australian property fund profile at September 2004 (PIR, 2004). Emerging sector funds were evident, with 54 funds accounting for 512 properties valued at

(1) Hotels have a significant history of being included in some major property funds in Australia; hence hotels were not considered as an emerging sector for this study. Similarly, residential property is typically not included in property funds in Australia, except for some property syndicates; hence residential property was also not considered as an emerging sector for this study. 
Table 5: Australian property sector funds profile ${ }^{(1)}$ : September 2004

\begin{tabular}{lccc}
\hline Sector & Number of funds & $\begin{array}{c}\text { Total assets of } \\
\text { funds }\end{array}$ & $\begin{array}{c}\text { Number of } \\
\text { properties }\end{array}$ \\
\hline Emerging sectors & 54 & $\$ 2.7 \mathrm{~B}$ & 512 \\
Healthcare & 12 & $\$ 0.4 \mathrm{~B}$ & 31 \\
Retirement & 10 & $\$ 0.3 \mathrm{~B}$ & 65 \\
Leisure & 8 & $\$ 1.0 \mathrm{~B}$ & 124 \\
Self-storage & 3 & $\$ 0.1 \mathrm{~B}$ & 22 \\
Childcare & 6 & $\$ 0.3 \mathrm{~B}$ & 240 \\
Carparking & 2 & $\$ 0.2 \mathrm{~B}$ & 9 \\
Agricultural & 13 & $\$ 0.4 \mathrm{~B}$ & 21 \\
& & & \\
Traditional sectors & 458 & $\$ 150.2 \mathrm{~B}$ & 2,725 \\
Office & 170 & $\$ 21.4 \mathrm{~B}$ & 367 \\
Retail & 116 & $\$ 53.3 \mathrm{~B}$ & 705 \\
Industrial & 58 & $\$ 7.3 \mathrm{~B}$ & 323 \\
Diversified & 88 & $\$ 65.8 \mathrm{~B}$ & 1,257 \\
Hotel & 26 & $\$ 2.4 \mathrm{~B}$ & 73 \\
\hline
\end{tabular}

Source: PIR (2004)

(1) Residential property funds (54 funds) and property development funds (11 funds) are not included

\$2.7 billion across seven emerging property sectors. The following sections provide an updated profile of the emerging property sector at October 2005.

\section{Self-storage}

Whilst traditionally being a sector for small investors and private companies (eg: Kennards, Millers, Storage King, National), self-storage has taken on increased importance in recent years, resulting from high density living, downsizing to smaller properties and businesses outsourcing their storage requirements. There are currently over 900 self-storage facilities in Australia, accounting for over 2 million $\mathrm{m}^{2}$ of storage space; occupancy rates are over $82 \%$, with the user profile being residential (75\%) and business (25\%) (Blackwell, 2005; Kennard and Blackwell, 2005; SSAA, 2005).

Recent years have seen increased institutional interest in self-storage (see Table 6A); particularly where the property fund has linked with an established operator in the selfstorage sector; for example:

APN with National

Valad with Kennards

Abacus with Storage King,
Mariner with Millers

Quantum with Storage King

with a range of operational business models used, including lease structures, joint ventures and stapled securities. 
Table 6A: Emerging property sector fund profile: October 2005

\begin{tabular}{|c|c|c|c|c|c|}
\hline Property fund & $\begin{array}{l}\text { Year } \\
\text { estab. }\end{array}$ & $\begin{array}{c}\text { Fund } \\
\text { type }\end{array}$ & $\begin{array}{l}\text { Number of } \\
\text { properties }\end{array}$ & $\begin{array}{c}\text { Total } \\
\text { assets }\end{array}$ & Fund manager \\
\hline \multicolumn{6}{|c|}{ Self-storage (8 funds @ \$408M) } \\
\hline $\begin{array}{l}\text { APN National } \\
\text { Storage PT }\end{array}$ & 2003 & URF & 20 & $\$ 134 \mathrm{M}$ & APN \\
\hline Mariner PT No. 2 & 2005 & URF & 1 & $\$ 29 \mathrm{M}$ & Mariner \\
\hline $\begin{array}{l}\text { Abacus Storage } \\
\text { Fund }\end{array}$ & 2005 & URF & 16 & $\$ 108 \mathrm{M}$ & Abacus \\
\hline $\begin{array}{l}\text { Valad Property } \\
\text { Group }\end{array}$ & 2004 & LPT & 24 & $\$ 114 \mathrm{M}$ & Valad \\
\hline $\begin{array}{l}\text { Storage King } \\
\text { Granville }\end{array}$ & 2001 & DPS & 1 & $\$ 4 \mathrm{M}$ & Quantum \\
\hline $\begin{array}{l}\text { Storage King } \\
\text { Chatswood }\end{array}$ & 1999 & DPS & 1 & $\$ 4 \mathrm{M}$ & Quantum \\
\hline $\begin{array}{l}\text { Storage King } \\
\text { St Peters }\end{array}$ & 2000 & DPS & 1 & $\$ 4 \mathrm{M}$ & Quantum \\
\hline $\begin{array}{l}\text { Storage King Lane } \\
\text { Cove }\end{array}$ & 1999 & DPS & 1 & $\$ 11 \mathrm{M}$ & Quantum \\
\hline \multicolumn{6}{|c|}{ Retirement (12 funds @ \$528M) } \\
\hline $\begin{array}{l}\text { ING Real Estate } \\
\text { Community Living } \\
\text { Fund }\end{array}$ & 2004 & LPT & 40 & $\$ 146 \mathrm{M}$ & ING \\
\hline $\begin{array}{l}\text { Prime Retirement \& } \\
\text { Aged Care PT }\end{array}$ & 2001 & URF & 20 & $\$ 166 \mathrm{M}$ & Aust. Prop. Custodian \\
\hline Village Life PT & 2003 & URF & 23 & $\$ 55 \mathrm{M}$ & Westpac \\
\hline Settlers Life PT & 2005 & URF & 3 & $\$ 27 \mathrm{M}$ & SAITeysMcMahon \\
\hline $\begin{array}{l}\text { APN Retirement } \\
\text { Properties Fund }\end{array}$ & 1999 & DPS & 5 & $\$ 32 \mathrm{M}$ & APN \\
\hline $\begin{array}{l}\text { Abacus Retirement } \\
\text { Living Trust }\end{array}$ & 2005 & DPS & 2 & $\$ 24 \mathrm{M}$ & Abacus \\
\hline $\begin{array}{l}\text { Forest Place } \\
\text { Clayfield } \\
\text { Syndicate }\end{array}$ & 1999 & DPS & 1 & $\$ 17 \mathrm{M}$ & FKP \\
\hline $\begin{array}{l}\text { Forest Place } \\
\text { Cleveland } \\
\text { Syndicate }\end{array}$ & 1999 & DPS & 1 & $\$ 8 \mathrm{M}$ & FKP \\
\hline $\begin{array}{l}\text { Sunraysia } \\
\text { Retirement Village } \\
\text { Syndicate }\end{array}$ & 1998 & DPS & 1 & $\$ 6 \mathrm{M}$ & Sunraysia \\
\hline $\begin{array}{l}\text { Grande Serviced } \\
\text { Apartments }\end{array}$ & 2000 & DPS & 1 & $\$ 27 \mathrm{M}$ & Becton \\
\hline $\begin{array}{l}\text { Syndicate No. } 6 \\
\text { Comptons }\end{array}$ & 1998 & STS & 1 & $\$ 15 M$ & SAITeysMcMahon \\
\hline $\begin{array}{l}\text { Farrington Grove } \\
\text { Retirement Estate }\end{array}$ & 2000 & DPD & 1 & $\$ 5 \mathrm{M}$ & William Buck \\
\hline
\end{tabular}

Pacific Rim Property Research Journal, Vol 12, No 2 
This currently sees 8 property funds involving self-storage, accounting for 65 properties valued at \$408 million. An unlisted retail fund is the general property vehicle used, with Valad having incorporated their 24 self-storage properties into the Valad Property Group (LPT) portfolio ( $>500$ million). Increased institutional involvement in this sector is expected as the sector consolidates and matures, although a lack of quality stock is a current concern.

In comparison, self-storage in the US has been more institutionalised in the last ten years (Severino, 2005), with over 41,000 self-storage facilities accounting for 1.65 billion $\mathrm{ft}^{2}$ (MiniCo, 2005). The largest organisations in US self-storage are Public Storage, Extra Space Storage, Shurgard Storage Centres, U-Haul International, U-Store-It Trust and Sovran Self-Storage, with five of these top six self-storage players being REITs, and the top 10 self-storage players accounting for over $16 \%$ of the market (MiniCo, 2005). Equivalent UK leaders in self-storage include Big Yellow, Lok'n Store and Safestone.

\section{Retirement}

Whilst retirement and aged care has traditionally been the domain of church and charity organisations, recent years have seen increased institutional involvement in this sector (see Table 6A). Key drivers have been an ageing population (ABS, 2004), with baby boomer wealth seeing increased expectations regarding retirement facility quality and services.

This currently sees 12 property funds involving retirement facilities, accounting for 99 retirement properties/villages valued at $\$ 528$ million. The most significant property vehicles are the ING Real Estate Community Living Fund, involving an LPT with \$146 million in assets in 40 Australian and US retirement facilities ${ }^{(2)}$, as well as the Prime Retirement \& Aged Care Property Trust (20 retirement facilities; \$166 million in assets) as an unlisted retail fund.

Further consolidation and expansion of the retirement sector is expected, particularly with the introduction of established property players diversifying into this sector and the establishment of partnerships between property fund managers and experienced developers in the retirement sector; eg: Primelife/Babcock \& Brown/MFS and FKP/Macquarie. Issues regarding the preferred management structure (eg: deferred management fee system) are currently being evaluated (Blundell, 2004).

\section{Childcare}

Childcare facilities have attracted recent attention from a range of property funds; largely unlisted retail funds (see Table 6B). This currently sees 7 property funds involving childcare facilities, accounting for 406 childcare facilities valued at $\$ 412$ million. The largest funds were Childcare Property Fund (\$146 million) and Australian Education Trust (LPT; $\$ 98$ million).

(2) ING Real Estate Community Living Fund also includes some student accommodation in New Zealand and US.

$186 \quad$ Pacific Rim Property Research Journal, Vol 12, No 2 
Table 6B: Emerging property sector fund profile: October 2005

\begin{tabular}{|c|c|c|c|c|c|}
\hline Property fund & $\begin{array}{l}\text { Year } \\
\text { estab. }\end{array}$ & $\begin{array}{l}\text { Fund } \\
\text { type }\end{array}$ & $\begin{array}{l}\text { Number of } \\
\text { properties }\end{array}$ & $\begin{array}{l}\text { Total } \\
\text { assets }\end{array}$ & Fund manager \\
\hline \multicolumn{6}{|c|}{ Childcare (7 funds @ \$412M) } \\
\hline $\begin{array}{l}\text { Australian Education } \\
\text { Trust }\end{array}$ & 2003 & LPT & 142 & $\$ 98 \mathrm{M}$ & Peppercorn \\
\hline $\begin{array}{l}\text { Australian Social } \\
\text { Infrastructure Fund }\end{array}$ & 2001 & URF & 61 & $\$ 94 \mathrm{M}$ & Ceramic \\
\hline $\begin{array}{l}\text { Childcare Property } \\
\text { Fund }\end{array}$ & 2004 & URF & 138 & $\$ 146 M$ & $\begin{array}{l}\text { SAITeysMcMah } \\
\text { on }\end{array}$ \\
\hline JF Childcare Fund & 2003 & URF & 31 & $\$ 27 \mathrm{M}$ & James Fielding \\
\hline Childcare PT No.1 & 2001 & URF & 9 & $\$ 14 M$ & DDH Graham \\
\hline Childcare PT No.2 & 2002 & URF & 11 & $\$ 14 \mathrm{M}$ & DDH Graham \\
\hline Childcare PT No.3 & 2002 & URF & 15 & $\$ 19 M$ & DDH Graham \\
\hline \multicolumn{6}{|c|}{ Leisure/Entertainment (13 funds @ \$1,569M) } \\
\hline $\begin{array}{l}\text { ING Real Estate } \\
\text { Entertainment Fund }\end{array}$ & 2004 & LPT & 15 & $\$ 176 \mathrm{M}$ & ING \\
\hline Macquarie Leisure & 1998 & LPT & 53 & $\$ 353 \mathrm{M}$ & Macquarie \\
\hline ALE Property Group & 2003 & LPT & 107 & $\$ 651 M$ & ALE \\
\hline $\begin{array}{l}\text { MFS Living and } \\
\text { Leisure Group }\end{array}$ & 1999 & LPT & 3 & $\$ 10 \mathrm{M}$ & MFS \\
\hline $\begin{array}{l}\text { MTM Entertainment } \\
\text { Trust }\end{array}$ & 1998 & LPT & 1 & $\$ 21 \mathrm{M}$ & $\begin{array}{l}\text { Babcock \& } \\
\text { Brown }\end{array}$ \\
\hline $\begin{array}{l}\text { Tourism \& Leisure } \\
\text { Trust }\end{array}$ & 1997 & LPT & 1 & $\$ 23 \mathrm{M}$ & James Fielding \\
\hline $\begin{array}{l}\text { Stadium Australia } \\
\text { Trust }\end{array}$ & 1997 & LPT & 1 & $\$ 164 M$ & James Fielding \\
\hline $\begin{array}{l}\text { Grant Samuel Laundy } \\
\text { Pub Fund }\end{array}$ & 2005 & URF & 4 & $\$ 70 \mathrm{M}$ & Grant Samuel \\
\hline $\begin{array}{l}\text { National Leisure and } \\
\text { Gaming }\end{array}$ & 2005 & LPT & 3 & $\$ 30 M$ & NLG \\
\hline JF Tourist Park Fund & 2004 & URF & 1 & $\$ 17 \mathrm{M}$ & James Fielding \\
\hline $\begin{array}{l}\text { Aspen Parks Property } \\
\text { Fund }\end{array}$ & 2004 & URF & 7 & $\$ 34 \mathrm{M}$ & Aspen \\
\hline $\begin{array}{l}\text { Abacus Mariners Cove } \\
\text { Trust }\end{array}$ & 2000 & DPS & 1 & $\$ 7 \mathrm{M}$ & Abacus \\
\hline $\begin{array}{l}\text { Warwick Cinema } \\
\text { Syndicate Trust }\end{array}$ & 1999 & DPS & 1 & $\$ 13 \mathrm{M}$ & Westpoint \\
\hline
\end{tabular}




\section{Leisure/entertainment}

The leisure and entertainment sector is the largest emerging markets sector (see Table 6B), with 13 property funds involving 198 properties valued at $\$ 1.6$ billion. A diverse range of leisure/entertainment property types are included, such as pubs, themeparks, marinas, bowling centres, theatres, caravan parks and tourist parks. The leisure/entertainment sector has made more substantial use of LPTs as the property investment vehicle, with Macquarie Leisure (\$353M), ALE Property Group (\$651M) and ING Real Estate Entertainment Fund $(\$ 176 \mathrm{M})$ being the most substantive vehicles. For example, Macquarie Leisure $(\$ 353 \mathrm{M})$ has a diverse portfolio comprising the Dreamworld theme park (53\% of portfolio), six marinas $(22 \%)$ and 46 bowling centres $(25 \%)$. Similarly, the ALE Property Group $(\$ 650 \mathrm{M})$ has a portfolio of 107 pubs (hotel assets of Fosters) leased to ALH on 25-year leases. The leisure/entertainment LPT sector has also seen strong investment performance in recent years; although higher risk was evident in delivering these higher returns; see next section for performance analysis.

\section{Healthcare}

Along with the retirement sector, healthcare will take on increased importance with an ageing population. While the traditional healthcare facilities sector has been dominated by private providers (eg: Mayne, Ramsay, Healthscope) (Blundell, 2003), larger property funds have become increasingly involved. Currently, there are 12 property funds involved in healthcare facilities, with 31 properties valued at $\$ 435 \mathrm{M}$; see Table $6 \mathrm{C}$. While property syndicates are the most widely-used property investment vehicle, the unlisted Australian Unity Healthcare Property Trust ( $\$ 179 \mathrm{M}$; 9 properties) is the largest healthcare facilities investment vehicle.

\section{Carparks}

The carpark sector involved 4 funds (see Table 6C), involving 11 carparks with assets of \$242M. The Sydney Opera House carpark comprises the Mariner Infrastructure Trust No.1 portfolio.

\section{Agriculture}

In the agriculture sector, 13 property funds (see Table $6 \mathrm{D}$ ) account for 57 properties valued at $\$ 650 \mathrm{M}$. These funds cover the areas of vineyards, almond plantations, beef cattle, cotton and horticulture. Whilst property syndicates have tended to be smaller single-property vehicles in agriculture, the more substantive property funds are the Challenger Wine Trust (LPT; \$281M; 34 vineyards) and the unlisted wholesale Colonial Agricultural Fund (\$208M; 8 beef cattle properties), with this unlisted wholesale fund typically used by superannuation funds seeking agricultural property exposure. 
Table 6C: Emerging property sector fund profile: October 2005

\begin{tabular}{|c|c|c|c|c|c|}
\hline Property fund & $\begin{array}{l}\text { Year } \\
\text { estab. }\end{array}$ & $\begin{array}{l}\text { Fund } \\
\text { type }\end{array}$ & $\begin{array}{l}\text { Number of } \\
\text { properties }\end{array}$ & $\begin{array}{l}\text { Total } \\
\text { assets }\end{array}$ & Fund manager \\
\hline \multicolumn{6}{|c|}{ Healthcare (12 funds @ \$435M) } \\
\hline $\begin{array}{l}\text { Australian Unity } \\
\text { Healthcare PT }\end{array}$ & 1999 & URF & 9 & $\$ 179 M$ & Australian Unity \\
\hline $\begin{array}{l}\text { Calan Healthcare } \\
\text { Aust. PT }\end{array}$ & 1998 & DPF & 3 & $\$ 59 M$ & Calan \\
\hline $\begin{array}{l}\text { Essential Health } \\
\text { Care Trust }\end{array}$ & 2002 & DPS & 3 & $\$ 56 \mathrm{M}$ & SAITeysMcMahon \\
\hline $\begin{array}{l}\text { Pacific Private } \\
\text { Property Trust }\end{array}$ & 2000 & DPS & 1 & $\$ 24 \mathrm{M}$ & SAITeysMcMahon \\
\hline PHC Darlinghurst & 2001 & DPS & 1 & $\$ 11 \mathrm{M}$ & SAITeysMcMahon \\
\hline $\begin{array}{l}\text { Sydney Healthcare } \\
\text { Trust }\end{array}$ & 2002 & DPS & 8 & $\$ 60 \mathrm{M}$ & SAITeysMcMahon \\
\hline $\begin{array}{l}\text { MAB Healthcare } \\
\text { Trust }\end{array}$ & 2003 & DPS & 1 & $\$ 15 M$ & MAB \\
\hline $\begin{array}{l}\text { MDRN Syndicate - } \\
\text { Medical No. } 1\end{array}$ & 1998 & DPS & 1 & $\$ 3 M$ & MDRN \\
\hline $\begin{array}{l}\text { Quantum PD } \\
\text { Syndicate No. } 6\end{array}$ & 2002 & DPS & 1 & $\$ 1 M$ & Quantum \\
\hline Holland Park & 1997 & DPS & 1 & $\$ 2 \mathrm{M}$ & SK Property \\
\hline $\begin{array}{l}\text { Havelock House } \\
\text { PT }\end{array}$ & 2002 & DPS & 1 & $\$ 21 \mathrm{M}$ & Becton \\
\hline $\begin{array}{l}\text { Merrylands } \\
\text { Property } \\
\text { Syndicate }\end{array}$ & 2000 & DPS & 1 & $\$ 4 M$ & Austgrowth \\
\hline \multicolumn{6}{|c|}{ Carparks (4 funds @ \$242M) } \\
\hline $\begin{array}{l}\text { Mariner } \\
\text { Infrastructure } \\
\text { Trust No. } 1\end{array}$ & 2004 & DPS & 1 & $\$ 90 \mathrm{M}$ & Mariner \\
\hline $\begin{array}{l}\text { International } \\
\text { Parking Group }\end{array}$ & 2003 & UWF & 8 & $\$ 135 \mathrm{M}$ & James Fielding \\
\hline $\begin{array}{l}\text { Macquarie St. Car } \\
\text { Park Fund }\end{array}$ & 2005 & DPS & 1 & $\$ 4 M$ & SAITeysMcMahon \\
\hline $\begin{array}{l}\text { Herston Road } \\
\text { Hospital Carpark } \\
\text { Property } \\
\text { Syndicate }\end{array}$ & 1998 & DPS & 1 & $\$ 13 \mathrm{M}$ & NHLS \\
\hline
\end{tabular}


Table 6D: Emerging property sector fund profile: October 2005

\begin{tabular}{|c|c|c|c|c|c|}
\hline Property fund & $\begin{array}{l}\text { Year } \\
\text { estab. }\end{array}$ & $\begin{array}{l}\text { Fund } \\
\text { type }{ }^{(1)}\end{array}$ & $\begin{array}{l}\text { Number of } \\
\text { properties }\end{array}$ & $\begin{array}{l}\text { Total } \\
\text { assets }\end{array}$ & Fund manager \\
\hline \multicolumn{6}{|c|}{ Agriculture (13 funds @ \$650M) } \\
\hline $\begin{array}{l}\text { Challenger Wine } \\
\text { Trust }\end{array}$ & 1999 & LPT & 34 & $\$ 281 \mathrm{M}$ & Challenger \\
\hline $\begin{array}{l}\text { Coonawarra Aust. } \\
\text { PT }\end{array}$ & 2003 & LPT & 1 & $\$ 16 \mathrm{M}$ & Coonawarra \\
\hline $\begin{array}{l}\text { Colonial } \\
\quad \text { Agricultural } \\
\text { Fund }\end{array}$ & 1997 & UWF & 8 & $\$ 208 M$ & Colonial FS \\
\hline $\begin{array}{l}\text { Primary } \\
\text { Infrastructure } \\
\text { Fund }\end{array}$ & 2004 & URF & 5 & $\$ 52 \mathrm{M}$ & SAITeysMcMahon \\
\hline $\begin{array}{l}\text { Conundrum } \\
\text { Vineyards Unit } \\
\text { Trust }\end{array}$ & 1994 & DPS & 1 & $\$ 18 \mathrm{M}$ & SAITeysMcMahon \\
\hline $\begin{array}{c}\text { Specific Vineyard } \\
\text { No. } 3 \text { Unit Trust }\end{array}$ & 2001 & DPS & 1 & $\$ 11 \mathrm{M}$ & SAITeysMcMahon \\
\hline $\begin{array}{l}\text { Howcroft Unit } \\
\text { Trust I }\end{array}$ & 1998 & DPS & 1 & $\$ 14 \mathrm{M}$ & SAITeysMcMahon \\
\hline $\begin{array}{l}\text { Howcroft Unit } \\
\text { Trust II }\end{array}$ & 1999 & DPS & 1 & $\$ 11 \mathrm{M}$ & SAITeysMcMahon \\
\hline $\begin{array}{l}\text { Treviso Table } \\
\text { Grape Unit Trust }\end{array}$ & 2002 & DPS & 1 & $\$ 7 \mathrm{M}$ & SAITeysMcMahon \\
\hline $\begin{array}{l}\text { Treviso Table } \\
\text { Grape Unit Trust } \\
\text { No. } 2\end{array}$ & 2003 & DPS & 1 & $\$ 11 \mathrm{M}$ & SAITeysMcMahon \\
\hline $\begin{array}{l}\text { Lake Powell } \\
\text { Almond Unit } \\
\text { Trust No. } 1\end{array}$ & 2004 & DPS & 1 & $\$ 14 \mathrm{M}$ & SAITeysMcMahon \\
\hline $\begin{array}{l}\text { Carina Park } \\
\text { Almond Unit } \\
\text { Trust }\end{array}$ & 1999 & DPS & 1 & $\$ 4 M$ & SAITeysMcMahon \\
\hline $\begin{array}{l}\text { Carina Park } \\
\text { Almond Unit } \\
\text { Trust } 2,3 \text { \& } 4\end{array}$ & 2001 & DPS & 1 & $\$ 3 M$ & SAITeysMcMahon \\
\hline
\end{tabular}




\section{EMERGING SECTOR PERFORMANCE ANALYSIS}

\section{Analysis of individual emerging sector LPTs}

At this stage, any performance analysis of the emerging property sectors needs to be based on the LPT market, as direct emerging property sector series are not available. ${ }^{(3)}$

At October 2005, there were nine emerging sector LPTs, covering the areas of:

- leisure/entertainment (6): Macquarie Leisure, ALE Property Group, ING Real Estate Entertainment, Tourism \& Leisure, MTM Entertainment, MFS Living and Leisure

- retirement (1): ING Real Estate Community Living

- childcare (1): Australian Education Trust

- agriculture (1): Challenger Wine Trust,

with a total market capitalisation of $\$ 1,078$ million, representing only $1 \%$ of the total LPT market capitalisation and only having two of these emerging sector LPTs included in the ASX 300 benchmark; namely Macquarie Leisure and Australian Education Trust.

Table 7 presents the risk-adjusted performance analysis over Nov. 2002-Oct. 2005 for each of these nine emerging sector LPTs, compared to a number of leading traditional sector LPTs with office, retail, industrial or diversified portfolios. Average annual returns are presented for one, three and five year periods, with not all emerging sector LPTs having been available for this full five-year period. Strong performance is clearly evident from Macquarie Leisure and the Tourism \& Leisure Trust, with the emerging sector LPTs typically having a larger annual risk than the traditional sector LPTs. On a risk-adjusted basis, both of these emerging sector LPTs (Macquarie Leisure and the Tourism \& Leisure Trust) are seen to significantly out-perform the traditional sector LPTs.

Whilst performance indices are available for a number of LPT sectors (UBS, 2005), including office, retail, industrial, diversified, international and stapled security LPTs, an equivalent performance index is not available for benchmarking emerging sector LPTs. As such, to assess the overall significance of the emerging sector LPTs, two emerging sector

(3) Emerging sector LPTs are not necessarily seen as a proxy for the direct emerging sectors, but represent the only public domain performance information currently available for the emerging property sectors. 
LPT performance indices were established using the five emerging sector LPTs ${ }^{(4)}$ available over this three year period of 2002-2005. These performance indices were:

- $\quad$ market cap-weighted emerging sector LPT index

- $\quad$ asset value-weighted emerging sector LPT index,

with Table 8 presenting the risk-adjusted performance analysis of the emerging sector LPTs, compared to the other LPT sectors and the overall stockmarket.

Table 7: Emerging sector LPT performance: October 2005

\begin{tabular}{|c|c|c|c|c|c|c|}
\hline \multirow{2}{*}{ LPT } & \multirow{2}{*}{$\begin{array}{c}\text { Market } \\
\text { cap } \\
\text { (@ Oct. } \\
2005)\end{array}$} & \multicolumn{3}{|c|}{ Average annual return ${ }^{(1)}$} & \multirow{2}{*}{$\begin{array}{c}\text { Annual } \\
\text { risk }^{(2)}\end{array}$} & \multirow{2}{*}{$\begin{array}{c}\text { Sharpe } \\
\text { index }\end{array}$} \\
\hline & & $1 Y$ & $3 Y$ & $5 Y$ & & \\
\hline \multicolumn{7}{|l|}{ Emerging sector LPTs } \\
\hline Macquarie Leisure & $\$ 354 M$ & $56.2 \%$ & $62.5 \%$ & $35.2 \%$ & $21.2 \%$ & 2.70 \\
\hline $\begin{array}{l}\text { Australian Education } \\
\text { Trust }\end{array}$ & $\$ 108 M$ & $0.0 \%$ & NA & NA & NA & NA \\
\hline ALE Property & $\$ 207 M$ & $44.5 \%$ & NA & NA & NA & NA \\
\hline $\begin{array}{l}\text { ING Real Estate } \\
\text { Community Living }\end{array}$ & $\$ 162 \mathrm{M}$ & $12.9 \%$ & NA & NA & NA & NA \\
\hline $\begin{array}{l}\text { ING Real Estate } \\
\text { Entertainment }\end{array}$ & $\$ 82 \mathrm{M}$ & $9.7 \%$ & NA & NA & NA & NA \\
\hline Tourism \& Leisure Trust & $\$ 19 M$ & $39.1 \%$ & $53.7 \%$ & NA & $26.9 \%$ & 1.80 \\
\hline MTM Entertainment Trust & $\$ 15 M$ & $30.4 \%$ & $14.9 \%$ & $13.4 \%$ & $47.8 \%$ & 0.20 \\
\hline $\begin{array}{l}\text { MFS Living \& Leisure } \\
\text { Group }\end{array}$ & $\$ 3 M$ & $-12.5 \%$ & $11.9 \%$ & NA & $119.4 \%$ & 0.06 \\
\hline Challenger Wine Trust & $\$ 128 \mathrm{M}$ & $-2.0 \%$ & $10.3 \%$ & NA & $10.1 \%$ & 0.50 \\
\hline \multicolumn{7}{|l|}{ Traditional sector LPTs } \\
\hline Stockland & $\$ 8.1 \mathrm{~B}$ & $10.9 \%$ & $17.5 \%$ & $17.9 \%$ & $12.7 \%$ & 0.96 \\
\hline GPT & $\$ 7.9 \mathrm{~B}$ & $9.7 \%$ & $17.9 \%$ & $16.2 \%$ & $14.2 \%$ & 0.89 \\
\hline Mirvac & $\$ 3.4 \mathrm{~B}$ & $-8.8 \%$ & $4.9 \%$ & $10.1 \%$ & $18.7 \%$ & -0.02 \\
\hline Centro & $\$ 4.6 \mathrm{~B}$ & $42.3 \%$ & $29.6 \%$ & $27.1 \%$ & $17.1 \%$ & 1.42 \\
\hline Macquarie CountryWide & $\$ 2.1 \mathrm{~B}$ & $9.7 \%$ & $15.6 \%$ & $15.8 \%$ & $11.4 \%$ & 0.90 \\
\hline Macquarie Office & $\$ 2.2 \mathrm{~B}$ & $8.8 \%$ & $9.1 \%$ & $11.4 \%$ & $9.0 \%$ & 0.42 \\
\hline ING Industrial & $\$ 1.8 \mathrm{~B}$ & $16.3 \%$ & $20.1 \%$ & $19.5 \%$ & $10.0 \%$ & 1.48 \\
\hline Macquarie Goodman & $\$ 5.2 \mathrm{~B}$ & $22.5 \%$ & $25.3 \%$ & NA & $15.2 \%$ & 1.32 \\
\hline
\end{tabular}

Source: UBS (2005)

(1) Not all emerging sector LPTs are available for full five-year period

(2) Annual risk is calculated as standard deviation of three year monthly returns

${ }^{(4)}$ Five emerging sector LPTs in the emerging sector LPT performance index are Macquarie Leisure, Tourism \& Leisure, MTM Entertainment, MFS Living and Leisure, and Challenger Wine.

$192 \quad$ Pacific Rim Property Research Journal, Vol 12, No 2 
Table 8: Australian emerging property sector LPT performance analysis: Nov. 2002 - Oct. 2005

\begin{tabular}{lccc}
\hline LPT sector & $\begin{array}{c}\text { Average } \\
\text { annual return }\end{array}$ & Annual risk & Sharpe index $^{(1)}$ \\
\hline $\begin{array}{l}\text { Emerging property LPTs } \\
\text { : market cap-weighted }\end{array}$ & $47.6 \%$ & $14.78 \%$ & $2.87(1)$ \\
$\begin{array}{l}\text { Emerging property LPTs: } \\
\text { asset value-weighted }\end{array}$ & $38.7 \%$ & $11.90 \%$ & $2.81(2)$ \\
Office LPTs & $9.7 \%$ & $9.90 \%$ & $0.45(10)$ \\
Retail LPTs & $18.1 \%$ & $8.90 \%$ & $1.44(3)$ \\
Industrial LPTs & $21.8 \%$ & $11.10 \%$ & $1.49(4)$ \\
Diversified LPTs & $13.7 \%$ & $12.30 \%$ & $0.68(8)$ \\
International LPTs & $12.2 \%$ & $9.40 \%$ & $0.74(7)$ \\
Stapled security LPTs & $11.9 \%$ & $9.90 \%$ & $0.67(9)$ \\
ASX LPT 300 & $15.5 \%$ & $8.20 \%$ & $1.25(6)$ \\
Shares & $18.5 \%$ & $8.80 \%$ & $1.50(3)$ \\
\hline
\end{tabular}

Source: Authors' calculations from UBS (2005)

(1) Ranks based on risk-adjusted performance are given in brackets.

\section{Analysis of emerging sector LPTs}

The emerging sector LPTs gave significantly higher average annual returns over 2002-2005 than any other LPT sector and the stockmarket, although the annual risk for the emerging sector LPTs was higher than for most other LPT sectors and for the stockmarket. On a riskadjusted basis (using the Sharpe index), the emerging sector LPTs significantly outperformed all other LPT sectors and the overall stockmarket. However, it should be recognised that this strong risk-adjusted performance by the emerging sector LPTs is significantly influenced by the high returns generated by Macquarie Leisure over this threeyear period, as well as the substantive weighting for Macquarie Leisure in the emerging sector market cap-weighted and asset value-weighted indices.

To assess the portfolio diversification benefits of the emerging sector LPTs, Table 9 presents the inter-asset correlation matrix for the various LPT sectors and the overall stockmarket. The emerging property sector LPTs were seen to be less correlated with the overall LPT sector $(r=0.32)$ than any of the other LPT sectors with the overall LPT sector $(r=0.66$ to 0.87$)$, reflecting enhanced within-LPT sector portfolio diversification benefits 
by the emerging sector LPTs, as well as the emerging sector LPTs being more correlated with the industrial LPT sector $(r=0.52)$ than with the office LPT sector $(r=0.24)$ and the retail LPT sector $(r=0.19)$. The emerging sector LPTs are not highly correlated with the overall stockmarket $(\mathrm{r}=0.41)$, with this correlation similar to that seen for each of the other LPT sectors with the overall stockmarket $(r=0.26$ to 0.42$)$. In interpreting these correlations, it is important to recognise that the emerging sector LPTs make up a small component of the overall LPT sector in Australia, with the larger LPT sub-sectors in the LPT index expected to be more highly correlated with the overall LPT sector.

Table 9: Australian LPT sector correlations: Nov. 2002 - Oct. 2005

\begin{tabular}{|c|c|c|c|c|c|c|c|}
\hline & $\begin{array}{l}\text { Emerging } \\
\text { LPTs }^{(1)}\end{array}$ & $\begin{array}{l}\text { Office } \\
\text { LPTs }\end{array}$ & $\begin{array}{l}\text { Retail } \\
\text { LPTs }\end{array}$ & $\begin{array}{l}\text { Industrial } \\
\text { LPTs }\end{array}$ & $\begin{array}{l}\text { Diversified } \\
\text { LPTs }\end{array}$ & $\begin{array}{l}\text { ASX } \\
\text { LPT } \\
300\end{array}$ & Shares \\
\hline $\begin{array}{l}\text { Emerging } \\
\text { LPTs }\end{array}$ & 1.00 & & & & & & \\
\hline Office LPTs & 0.24 & 1.00 & & & & & \\
\hline Retail LPTs & 0.19 & $0.43^{\star}$ & 1.00 & & & & \\
\hline $\begin{array}{l}\text { Industrial } \\
\text { LPTs }\end{array}$ & $0.52^{*}$ & $0.70^{*}$ & $0.36^{*}$ & 1.00 & & & \\
\hline $\begin{array}{l}\text { Diversified } \\
\text { LPTs }\end{array}$ & 0.21 & $0.73^{*}$ & $0.40^{*}$ & $0.54^{\star}$ & 1.00 & & \\
\hline $\begin{array}{l}\text { ASX LPT } \\
300\end{array}$ & 0.32 & $0.80^{*}$ & $0.77^{*}$ & $0.66^{\star}$ & $0.87^{*}$ & 1.00 & \\
\hline Shares & $0.41^{*}$ & $0.42^{\star}$ & $0.33^{*}$ & $0.36^{*}$ & 0.26 & $0.40^{*}$ & 1.00 \\
\hline
\end{tabular}

*: Significant correlation $(\mathrm{P}<5 \%)$

(1) Correlations are only presented using market cap-weighted emerging sector LPT index to be consistent with other market cap-weighted LPT indices

Overall, these empirical results for the emerging sector LPTs confirm the strong riskadjusted performance by the emerging sector LPTs over this 2002-2005 period and the portfolio diversification benefits available from these emerging sector LPTs.

However, a key on-going strategic issue regarding the emerging property sectors in Australia is whether this out-performance can be sustained. Whilst the emerging property sectors are coming off a low base and property funds have been able to identify quality property assets and strategic alliances to manage the business risk component in this sector, the ability to continue this momentum will place considerable focus on ongoing property asset selection and property fund manager expertise. For example, the size of the Australian market in these emerging sectors is seeing some emerging sector property funds already including international property in their emerging property sector portfolios (eg: ING Real Estate Community Living Fund). This ability to deliver on-going out-performance will also 
vary across different emerging property sectors. For example, the retirement and healthcare sectors will be sustained by the changing demographics in Australia, whereas the leisure/entertainment sectors will be more influenced by economic growth, with risk factors such as increasing interest rates and potential reduced disposable income being key factors for the leisure/entertainment sectors.

\section{CONCLUSION}

Capital inflow into property funds has seen increased competition for quality local properties, as well as increased levels of international property in property portfolios. It has also recently seen property funds seeking opportunities for enhanced returns from the emerging property sectors such as self-storage, healthcare, retirement, carparks and leisure/entertainment properties.

Whilst some of these emerging property sectors are small niche markets in Australia (Blundell, 2005b) and their longer-term benefits are yet to be fully assessed (Blundell, 2004), property funds are now considering the added-value that these emerging property sectors may bring to property portfolios. Issues that will have a key role in expanding the stature of these emerging property sectors are:

- $\quad$ increased market depth and maturity with further listed and unlisted vehicles

- increased investor confidence and experience in this sector

- development of direct property performance series for the emerging property sectors (eg:IPD/PCA series)

- more substantive emerging sector performance analysis, including a longer timeframe and an increased number of emerging sector funds

- developing suitable business models re: operating business (eg: retirement)

- more major property players effectively incorporating these emerging sectors into their property portfolio strategy; eg: Stockland and retirement properties

- establishing suitable joint ventures between property fund and development partners (eg: Primelife, Babcock \& Brown and MFS re: retirement properties) and business operating units (eg: self-storage)

- potential consideration of other emerging property sectors such as golf resorts (eg: OFM Resort and Leisure Trust) and infrastructure (eg: James Fielding Infrastructure Fund),

with the emerging property sectors expected to take on increased importance in Australia as property funds seek future local opportunities for enhanced property portfolio performance. The risk-adjusted performance analysis for emerging sector LPTs has also clearly demonstrated its contribution and portfolio diversification benefits to an investment portfolio over 2002-2005, with this needed to be further assessed over longer timeframes to more fully assess the strategic contribution of the emerging property sector in a property portfolio. 


\section{REFERENCES}

Australian Bureau of Statistics (2004), ABS Census of Population and Housing and Population Projections (\#3222.0). ABS, Canberra.

Blackwell, D. (2005), Understanding self storage. API CPD seminar, Sydney.

Blundell, L. (2003), Popping the healthcare pill. Property Australia 18 (1): 38-41.

Blundell, L. (2004), Survival tactics. Property Australia 18 (5): 26-28.

Blundell, L. (2005a), Sparking up change. Property Australia 19 (9): 36-39.

Blundell, L. (2005b), Time to step outside the comfort zone. Property Australia 20 (1): 1820.

Blundell, L. (2005c), Casting a bigger shadow. Property Australia 20 (2): 34-37.

DB RREEF (2005), The issues in managing a real estate portfolio. DB RREEF: Sydney.

Higgins, D. (2005), Modelling the Australian property investment universe: a preliminary study. Pacific Rim Property Research Journal 11: 268-281.

Kennard, S. and Blackwell, D. (2005), News from Australia: self-storage in the land down under. Mini Storage Messenger: October.

Larsen, C. (2002), Finding the old money. Property Australia 17 (4): 6-8.

Larsen, C. (2005), The Big Think: predicting the future for property. Property Australia 20 (1): 54-55.

Lowrey, C. (2005), Niche strategies in the current market. Prudential Real Estate Investors, Parsippany.

MiniCo (2005), 2006 Self Storage Almanac. MiniCo, Phoenix.

Murdoch, J. (2004), The globalisation of Australian LPTs. Australian Property Journal 38(1): 5-12.

NAREIT (2005a), NAREIT Real Estate Chartbook: October 2005. NAREIT, Chicago.

NAREIT (2005b), REITWatch: October 2005. NAREIT, Chicago.

Norris, S. (2004), Exploring the LPT landscape. Property Australia 18 (9): 54-56. 
Oliver, S. (2004), LPT changes - implications for investors. Property Australia 19(1): 106107.

PIR (2004), Australian Property Funds Industry Survey 2004. PIR, Melbourne.

Schuck, E. and Howard, J. (2005), Implementing an allocation to direct property. Australian Property Journal 38 (7): 535-541.

Self Storage Association of Australasia (2005), Australasian Self Storage Almanac. SSAA, Kilmore.

Severino, R. (2005), The US self-storage market. Prudential Real Estate Investors, Parsippany.

Tan, Y. K. (2004a), Benchmarking international property in Australian LPT portfolios. Pacific Rim Property Research Journal 10 (1): 3-29.

Tan, Y. K. (2004b), The role of international property trusts in Australian mixed-asset portfolios. Pacific Rim Property Research Journal 10 (2): 215-234.

Tan, Y. K. (2004c), Is development good for LPTs? Property Australia 19 (3): 50-51.

UBS (2005), UBS Indices: October 2005. UBS, Sydney. 